\title{
ELEKTRONENMIKROSKOPISCHE UNTERSUCHUNGEN ZUM PARASITENBEFALL DER MALPIGHISCHEN GEFÄSSE VON APIS MELLIFERA DURCH MALPIGHAMOEBA MELLIFICAE (RHIZOPODA)
}

\author{
Ulrich SCHWANTES und Dieter EICHELBERG * \\ Institut für Allgemeine und Spezielle Zoologie der Justus-Liebig-Universität Giessen. \\ Stephanstrasse 24, D-6300 Giessen, Bundesrepublik Deutschland
}

\section{ZUSAMMENFASSUNG}

Untersucht wurde die Feinstruktur der Malpighischen Gefäße der Honigbiene Apis mellifera vor und nach dem Befall durch Malpighamoeba mellificae. Die Epithelzellen dieser ungegliederten Gefäße zeigen einen für transportaktive Gewebe typischen Aufbau. Nach der Infektion mit $M$. mellificae verändert sich die Struktur der Zellen: die wesentlichen Veränderungen bestehen in einer Abflachung der Zellen, Verkümmerung des Mikrovillisaumes sowie einer starken Abnahme der Vesikulation. Die Schädigung der Bienen durch $M$. mellificae scheint offensichtlich in einer Nierenfunktionsstörung $\mathrm{zu}$ bestehen. In den Malpighischen Gefäßen wurden an Entwicklungsstadien die amoeboide Kriechform, Praecysten und Dauercysten beschrieben.

\section{EINLEITUNG}

Die Honigbiene Apis mellifera $L$. ist ein Insekt von großer ökologischer und ökonomischer Bedeutung. Es ist daher verständlich, daß auch das Studium ihrer Parasiten stets großes Interesse gefunden hat. Als Parasiten der Biene sind auch Protozoen bedeutsam, wobei hier vor allem Nosema apis (Sporozoa; Microsporidia) und Malpighamoeba mellificae (Rhizopoda) genannt sein sollen. Beide Parasiten treten häufig als Doppelinfektion auf (Schulz-Langner, 1957 ; Giordani, 1959 ; BorChert, 1966 ; CANTwell, 1974). Lange Zeit galt die nur im Darm parasitierende Nosema apis als der gefährlichere Parasit. Durch die medikamentöse Bekämpfungsmöglichkeit der Nosematose (z.B. Fumidil) ist ihre Gefährlichkeit allerdings stark gemindert worden. Die Verluste im Bienenvolk durch den Befall

\footnotetext{
* Mit dankenswerter Unterstützung durch die Deutsche Forschungsgemeinschaft.
} 
mit Malpighamoeba mellificae scheinen heute daher vom parasitologischen Standpunkt bedeutsamer. $M$. mellificae wurde erstmals von MAASSEN (1919) beschrieben und von Prell klassifiziert (1926, 1927). Die Amoeben parasitieren in den Malpighischen Gefäßen, insbesondere der Arbeiterinnen (Schulz-Langner, 1969).

Die 80 bis über 100 Malpighischen Gefäße der Honigbiene sind - wie bei allen Insekten - wesentlich an der Exkretion und Osmoregulation beteiligt. Diese schlauchförmigen Organe münden am Übergang vom Mitteldarm zum Enddarm, in den sie ihre Exkrete entleeren.

Über die Schäden, die der Parasit an den befallenen Organen bewirkt, liegen bisher noch keine elektronenmikroskopischen Befunde vor. Diese Lücke versucht unsere Studie zu schließen.

\section{METHODIK UND MATERIAL}

Die infizierten Bienen entstammten einem Bienenvolk aus Krofdorf-Gleiberg (Kreis Gießen). Sie wurden in der zweiten Aprilhälfte dem Volk entnommen. Bei der Infektion der Tiere handelt es sich um eine reine Amoebeninfektion. Die Praeparation des Darmtraktes erfolgte nach Immobilisieren der Tiere durch Kälte. Für die Lichtmikroskopie wurde in Bouinschem Gemisch fixiert, die Färbung mit Azan und Haematoxylin-Eosin durchgeführt. Außerdem wurden mit Toluidinblau angefärbte Semidünnschnitte angefertigt.

Für die Elektronenmikroskopie wurde für die Fixierung ein $1: 1$ Gemisch von Kaliumbichromat (1\%) und Osmiumtetroxid $(1 \%$ ) mit einem Zusatz von $0,7 \% \mathrm{NaCl}$ verwendet (WoHLFAHRTBottermanN, 1957). Der pH-Wert der Lösung wurde auf 7,1-7,2 eingestellt. Dic Entwässerung erfolgte über mehrere Acetonstufen, wobei in $70 \%$ igem Aceton zwei Stunden mit einem Gemisch von $1 \%$ iger Phosphorwolframsäure und $1 \%$ igem Uranylacetat nachkontrastiert wurde. Ultradünnschnitte wurden an einem Ultratom $11 \mathrm{I}$ (LKB), die EM-Aufnahmen mit einem Zeiss EM 9 S-2 und am EM 300, Philips, angefertigt.

\section{ERGEBNISSE}

\section{Die Strukturen nicht parasitierter Malpighischer Gefäße}

Um die eventuell auftretenden Veränderungen an den Nierenorganen nach Befall durch $M$. mellificae erkennen und deuten zu können, ist die Kenntnis des normalen Aufbaus der Gefäße Voraussetzung. Die Beschreibung der Normalmorphologie wird daher zunächst gegeben.

Die blindendenden, unverzweigten Malpighischen Gefäße (MG) liegen frei in der Leibeshöhle. Sie werden in ihrer gesamten Länge von mehreren von Tracheen 
versorgten Muskelbändern spiralig umschlossen, deren Kontraktionen eine Beweglichkeit der Gefäße in der Haemolymphe ermöglichen. Der Durchmesser der Gefäße nimmt zur Mündung hin zu ; er schwankt zwischen 40 und $100 \mu$. Diese Tatsache spiegelt sich auch in der Anzahl der das Lumen umschließenden Zellen wider. Im distalen Bereich der Gefäße sind es 3-4 Zellen, im proximalen Bereich sind es hingegen 9-12 (Abb. 1 a). Dem einschichtigen Epithel der Organe ist zur Leibeshöhle hin eine Basalmembran aufgelagert, welche gleichzeitig die Epithelzellen mit den Muskelbändern verbindet.

Das Epithel der imaginalen MG wird von zwei unterschiedlichen Zelltypen aufgebaut. In Anlehnung an Befunde bei anderen Insektenarten (Calliphora : BerRidge und OSCHMAN, 1969 ; Ephydra : EiCHELBERG, 1979 ; Drosophila : Wessing und Eichelberg, 1978) werden im folgenden diese beiden Zelltypen als Haupt- und Nebenzellen bezeichnet. Die Hauptzellen sind der wesentlich prominentere Zelltyp, sie sind bedeutend größer a!s die Nebenzellen. Der zweitc Zelltyp ist in weitaus geringerem Maße anzutreffen. Diese kleineren Zellen liegen vereinzelt zwischen den Hauptzellen (Abb. 1 a).

Die elektronenmikroskopischen Aufnahmen der Hauptzellen zeigen das typische Bild transportaktiver Zellen. Stark ausgebildet sind bei ihnen dic extrazellulären Spalträume des basalen Labyrinths, deren Ausläufer teilweise sehr weit in die einzelnen Zellen vordringen. Im Bereich der basalen Zelleinfaltungen, und mit deren Membranen eng assoziiert, finden sich viele Mitochondrien. In der intermediären Region der Zellen treten Mitochondrien nur vereinzelt auf, während sie in der Region des Zellapex wieder vermehrt zu finden sind. In der Intermediärregion ist der Zellkern gelegen. Das Karyoplasma läßt Regionen stärkerer und schwacher Elektronendurchlässigkeit erkennen, die die im Interphasekern vorliegenden unterschiedlichen Chromatinarten widerspiegeln : aufgrund der unterschiedlichen Anfärbbarkeit erscheint das kondensierte, stoffwechselinaktive Heterochromatin kontrastreicher als die stoffwechselaktive Form, das Euchromatin. Besonders kontrastreich stellt sich der Nucleolus dar, der oft in mehreren Anschnitten zu erkennen ist (Abb. 1 b).

In großer Anzahl sind im Cytoplasma der Hauptzellen elektronenlichte Vakuolen zu erkennen. Im basalen Bereich liegen sie gehäuft an den Einfaltungsräumen des Labyrinths.

Zum Gefäßlumen hin nimmt die Zahl der Vesikel ab, ihre Größe dagegen zu. Neben diesen elektronendurchlässigen offensichtlichen Transportvesikeln treten in geringerer Zahl elektronendichte Vesikel (Pigment ?) und Vakuolen mit konzentrischen Ringstrukturen sowie lipiderfüllte Vakuolen auf.

Ein dichtgedrängt stehender Saum von Mikrovilli bildet den zum Gefäßlumen hin gerichteten apikalen AbschluB der Hauptzellen; hierdurch wird die 


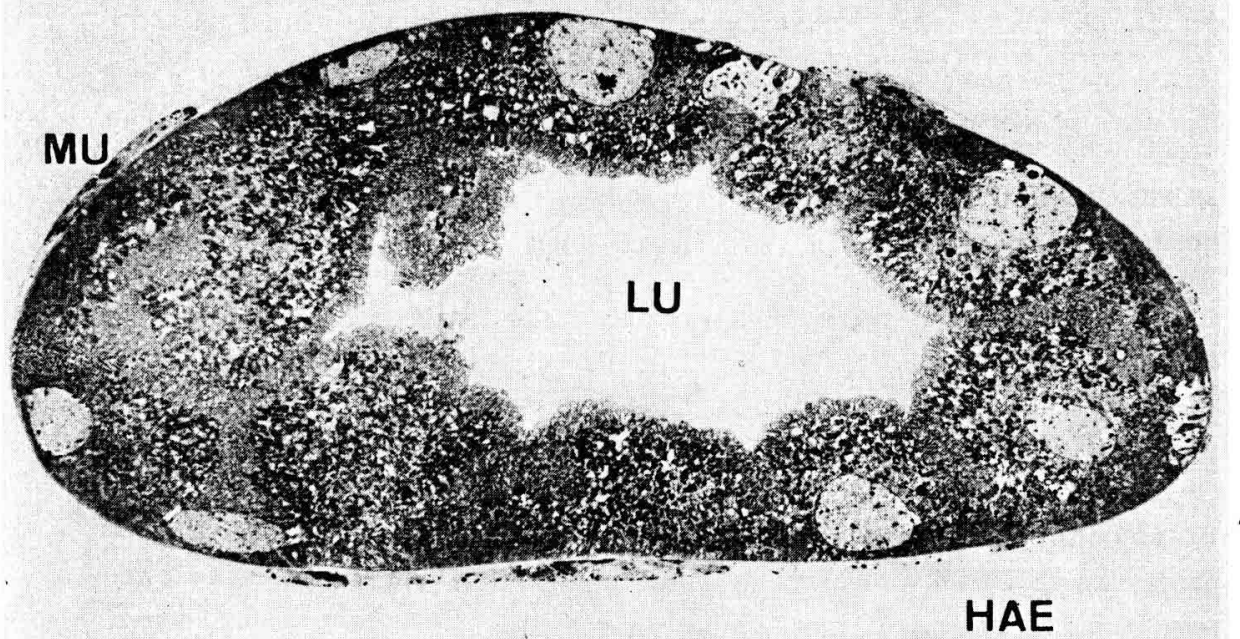

a
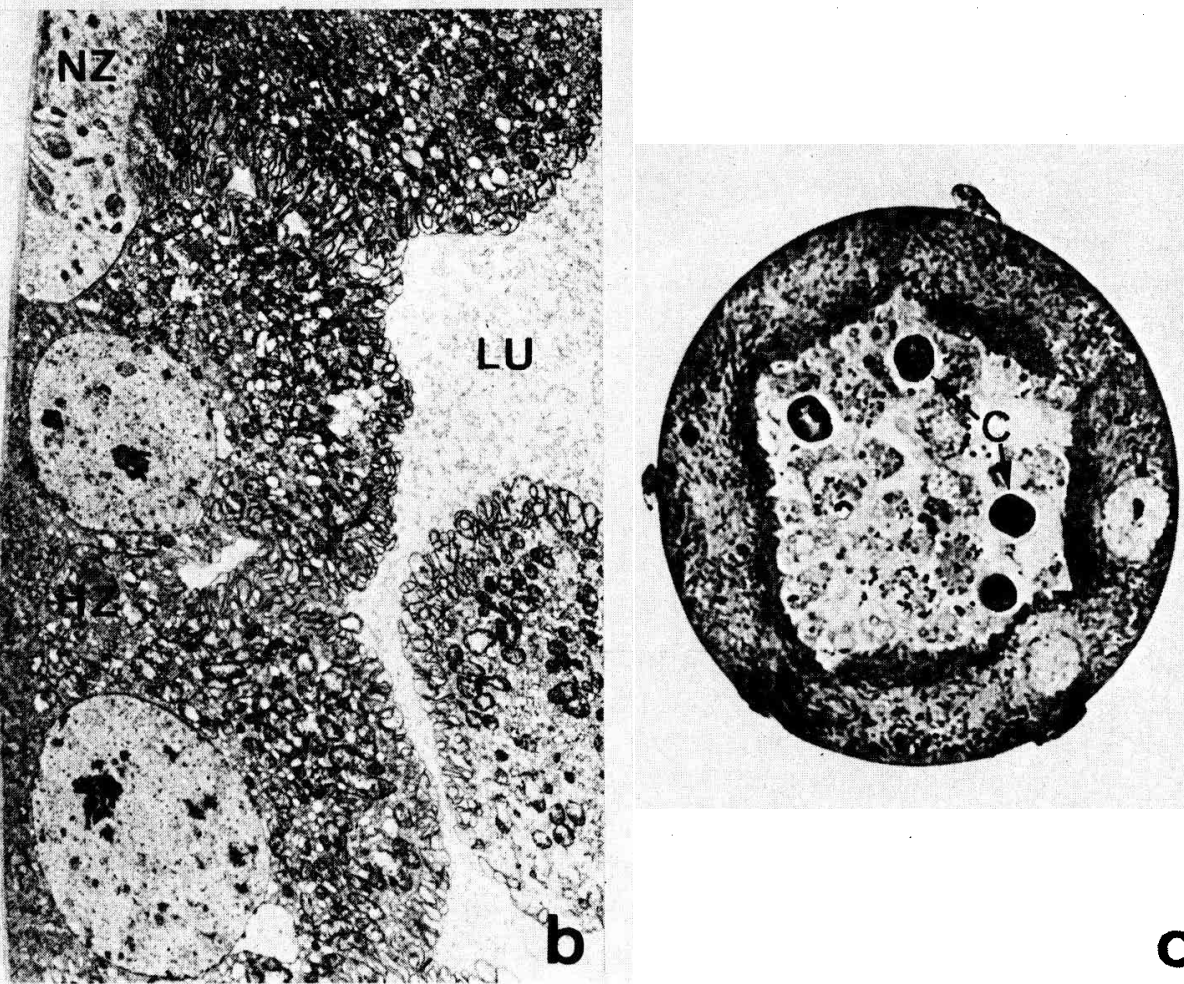

ABB. 1 
apikale Membranfläche der Zelle stark vergrößert (Abb. 1 a + b). In die Mikrovilli ziehen gelegentlich Ausläufer des endoplasmatischen Retikulums. Auffallend ist schließlich, daß die Hauptzellen sehr stark in ihrer Höhe variieren. So ragen einzelne Zellen regelrecht aus dem Zellverband heraus. Durch derartige Vorwölbungen kommt es zu einer zusätzlichen Vergrößerung der apikalen Membranfläche.

Die Nebenzellen unterscheiden sich in ihrem Aufbau deutlich von den Hauptzellen. Sie sind erheblich flacher als jene und besitzen lange Zellausläufer, die sich weit zwischen die benachbarten Hauptzellen schieben. Auf den elektronenmikroskopischen Bildern sind daher häufig nur Anschnitte dieser Zellen zu erkennen (Abb. $1 \mathrm{a}+\mathrm{b}$ ).

Ein basales Labyrinth ist bei ihnen nur spärlich ausgebildet. Mitochondrien sind in geringerer Menge anzutreffen, ihre Lage läßt keine polare Ausrichtung erkennen. Die Mitochondrien sind hier auch schmaler und kürzer als in den Hauptzellen. Das Cytoplasma der Nebenzellen ist kontrastarm, und Transportvesikel fehlen hier fast völlig. Ebenfalls fehlen diesen Zellen die für die Hauptzellen beschriebenen kontrastreichen «Pigment-Vakuolen».

Als apikalen Abschluß zum Gefäßlumen besitzen die Nebenzellen ebenfalls einen Mikrovillisaum, wobei diese Mikrovilli allerdings wesentlich kürzer sind als die der Hauptzellen.

\section{Die Strukturen parasitierter Malpighischer Gefäße}

Im Gegensatz zu den fast durchscheinenden Organen nicht infizierter Bienen sind die Malpighischen Gefäße amoebeninfizierter Tiere von milchigweißem Aussehen und wirken gelegentlich etwas aufgetrieben. Die Epithelzellen erscheinen stark abgeflacht. Die für die gesunden Gefäße beschriebene charakteristische Faltenbildung aufgrund unterschiedlicher Zellgrößen, entfällt bei den infizierten Organen. Das hierdurch stark erweiterte Gefäßlumen ist fast überall dicht mit den unterschiedlichen Entwicklungsstadien von $M$. mellificae erfüllt (Abb. $1 \mathrm{c}$ ). Insbesondere sind hier die amoeboide, geißellose Kriechform sowic Praecysten und Dauercysten zu finden. Auffallend ist, daß auf den elektronenmikroskopischen

Aвв. 1. а. - Querschnit durch den proximalen Bereich eines Malpighischen Gefäßes $(\times 1400)$.

b. - Epithelzellen eines Nierentubulus $(\times 3200)$.

c. - Semidimnschnitt durch einen mit Malpighamoeba mellificae infizierten Nierentubulus

(X 1200$)$. C - Cyste, HAE - Haemolymphe, HZ - Hauptzelle, LU - Gefäßlumen, MU - Muskelfaser, NZ - Nebenzelle.

Fig. 1. a. - Transverse section of the proximal region of a Malpighian tubule ( $\times 1400)$. b. - Epithelial cell of a Malpighian tubule ( $\times 3200)$.

c. - Transverse section of a Malpighian tubule infected by Malpighamoeba mellificae ( $\times 1200)$.

C - cyst, HAE - hemolymph, HZ - primary cell, LU - lumen, MU - muscular fibre. 

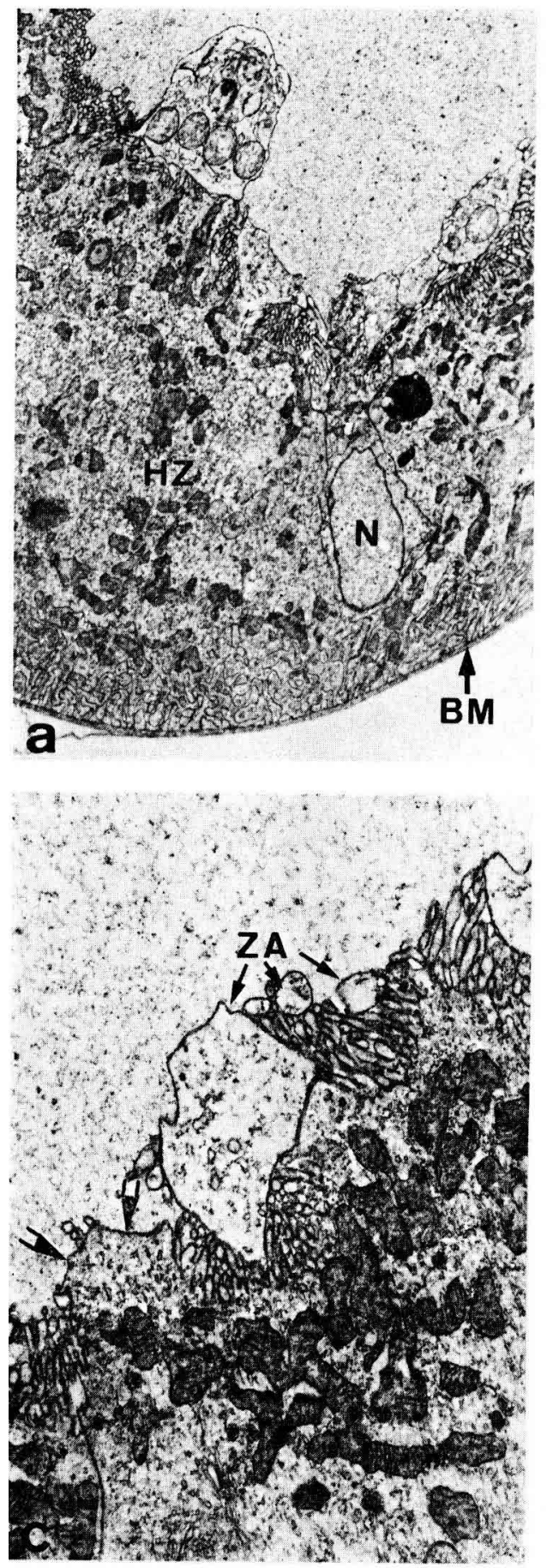
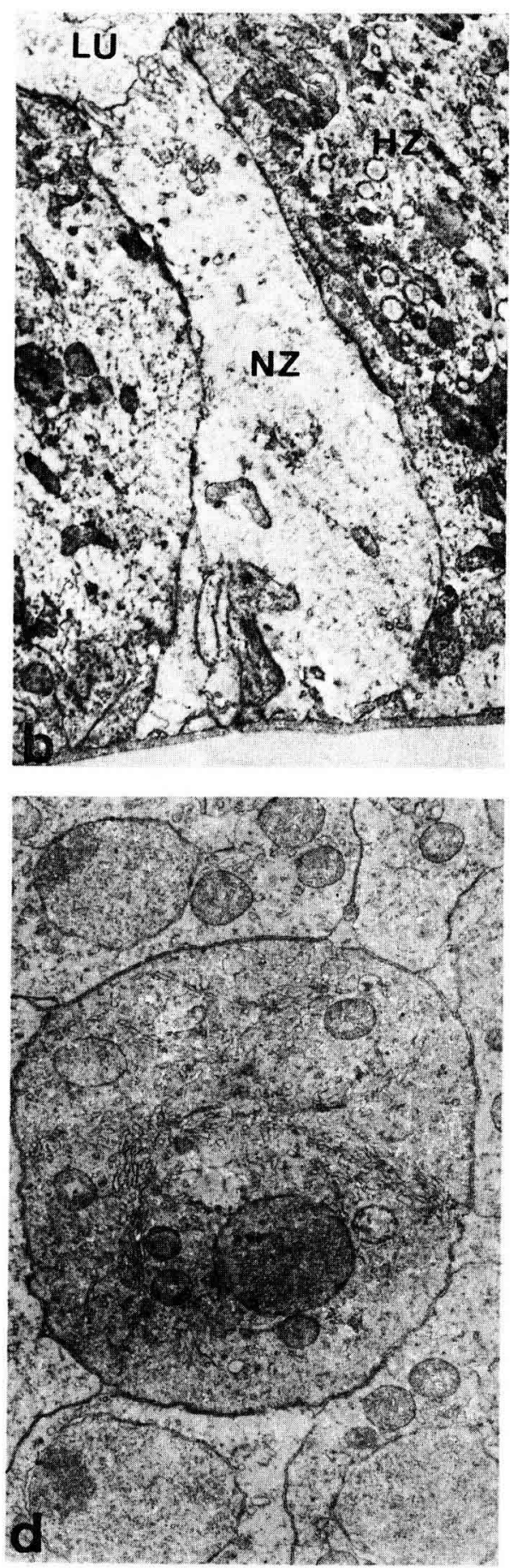
Aufnahmen die Abflachungen der Epithelzellen auch in jenen Regionen der Gefäße auftreten, in denen das Lumen nicht prall mit Amoeben erfüllt ist.

Die feinstrukturellen Veränderungen nach $M$. mellificae-Infektion betreffen insbesondere die intermediäre und apikale Zellregion (Abb. 2 a). Die Basalmembran, die basalen extrazellulären Einfaltungsräume, die Größe, Anzahl und Lage der Mitochondrien sind fast unverändert. Gleiches gilt für den Zellkern. Das endoplasmatische Retikulum und die Golgikomplexe erscheinen eher verstärkt. Die elektronenlichten Transportvesikel dagegen fehlen den Zellen dieser Gefäße fast vollkommen. Gleiches trifft für die Lipidvakuolen zu.

Dic auffälligsten Veränderungen der Zellen betreffen jedoch den apikalen Bürstensaum. Seine Mikrovilli liegen in den verbleibenden geringen Freiräumen zusammengedrängt, und sie sind zudem kürzer als die gesunder Gefäße. Im apikalen Membranbereich treten jetzt sogar kurze Abschnitte auf, denen ein Mikrovillibesatz völlig fehlt. Anstelle eines Mikrovillisaumes grenzt hier eine nicht gefaltete Zellmembran die Zellen zum Lumen ab (Abb. $1 \mathrm{~d}$ ).

\section{Die Strukturen von Malpighamoeba mellificae.}

In den untersuchten Gefäßen konnten wir die amoeboide Kriechform, sowie Prae- und Dauercysten von $M$. mellificae finden, begeißelte flagellate Formen (Schulz-Langner, 1961, 1964) traten nicht auf. Die amoeboiden Formen sind häufig so dicht gelagert, daß der Eindruck eines epithelialen Verbandes entsteht, der fast das gesamte Lumen erfüllt (Abb. 1c, $2 \mathrm{c}+3$ ). In den schwächer besiedelten Gefäßabschnitten liegen alle Malpighamoeben dem Mikrovillisaum der Epithelzellen an (Abb. $1 \mathrm{a}+\mathrm{d}$ ). Oft dringen dabei ihre lappigen Pseudo-

Авв. 2. a-c. - Anschnitte eines schwach infizierten Malpighischen Gefäßes.

a. - Geschüdigte Hauptzelle, Transportvesikel fehlen $(\times 5700)$.

b. - Hauptzellen und stark veränderte Nebenzelle ( $\times 8700)$.

c. - Anschnitte von Zellausläufern von Malpighamoeba mellificae am Apex der Tubuluszelle, deren Mikrovillisaum stellenweise stark reduziert ist (Doppelpfeil) $(\times 12200)$.

d. - Praecyste yon Malpighamoeba mellificae im Lumen eines stark besiedelten Nierengefäßes ( $\times 10000)$. BM - Basalmembran, HZ - Hauptzelle, LU - Gefäßlumen, N - Nucleus, NZ - Nebenzelle, ZA - Zellausläufer.

Fig. 2. a.-c. - Section of a slightly infected Malpighian tubule.

a. - Injured primary cell without transport vesicles ( $\times 5700)$.

b. - Primary cell and a strongly allered secondary cell $(\times 8700)$.

c. - IIigh magnification of a pseudopodia of Malpighamoeba mellificae at the apex of $a$ tubular cell. The brush border is significantly reduced in size (large arrows) ( $\times 12200)$.

d. - Pre-cyst of Malpighamocba mellificae in the lumen of a strongly infected malpighian tubule ( $\times 10000)$. BM - basement membrane, HZ - primary cell, LU - lumen, $\mathrm{N}$ - nucleus, NZ - secondary cell, ZA - pseudopodia. 
podien in den Mikrovillisaum ein. Die Zellmembranen der Amoeben liegen dabei der apikalen Membran der Wirtszelle eng an. Das periphere Cytoplasma (Ektoplasma) enthält kaum membranöse Einschlüsse, Organellen sind auf den endoplasmatischen Bereich begrenzt. Die meist runden Mitochondrien von $M$. mellificae sind vom Tubulus-Typ.

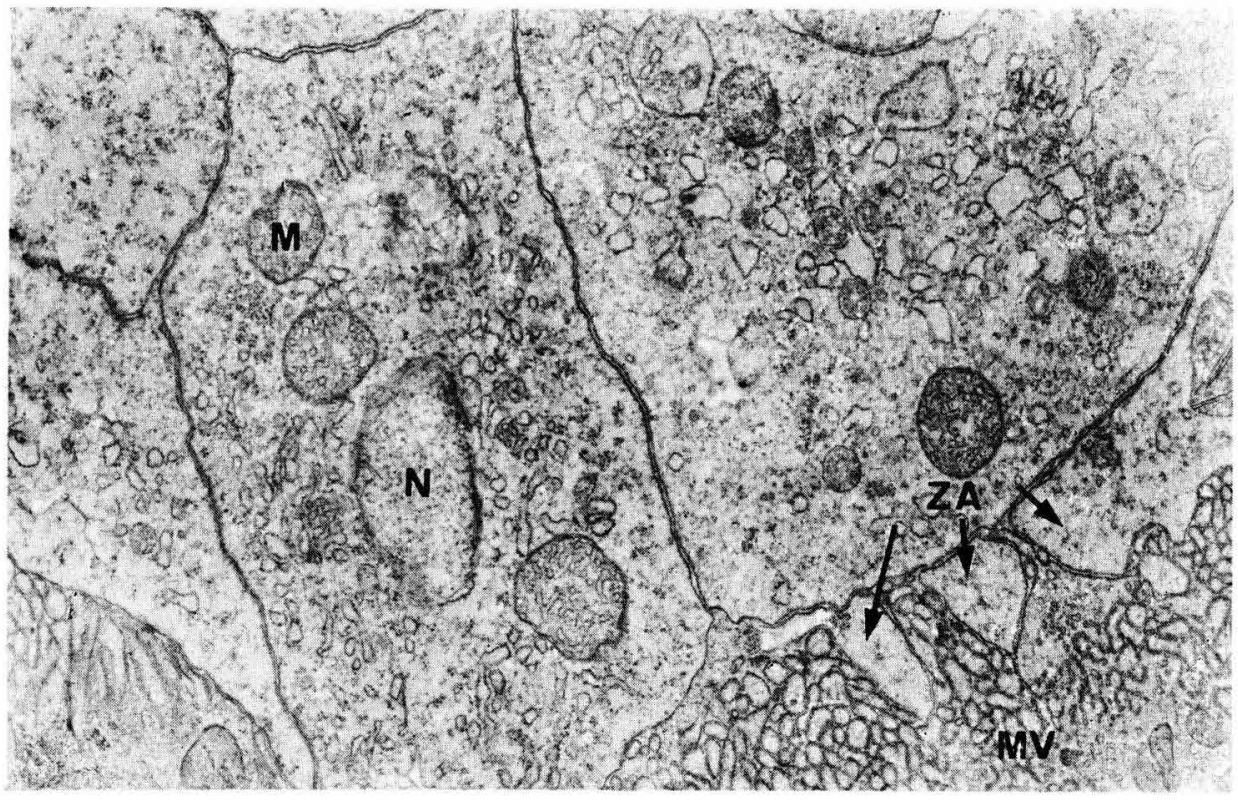

Авв. 3. Dichtgelagerte Malpighamoeben aus dem Lumen eines stark infizierten Nierengefäßes von Apis mellifera, die einen epithelialen Verband bilden ( $\times 14500)$. M - Mitochondrium, MV - Mikrovilli, N - Nucleus, ZA - Zellausläufer.

FIG. 3. Strongly infected Malpighian tubule of Apis mellifera showing amoebae lying close together as a pseudo-epithelium ( $\times 14500)$.

M - mitochondrion, MV - microvillus, N - nucleus, ZA - pscudopodia.

Der perinucleäre Raum ist stellenweise stark erweitert. Das Karyoplasma erscheint fast gleichmäßig granulär strukturiert, randständige Bereiche zeigen häufig einen verstärkten Kontrast.

Im Endoplasma liegen weiterhin auffallend viele Vakuolen und endoplasmatisches Retikulum. Golgikomplexe sind auf den Bildern nur gelegentlich $\mathrm{zu}$ erkennen.

Neben den bereits erwähnten, elektronendurchlässigen Vakuolen finden sich in einigen Amoebenzellen größere membranumhüllte Areale. Ihre Form und 
Größe sind unterschiedlich, sie können annähernd rund, jedoch auch unregelmäßig gestaltet sein. Im Inneren dieser Vakuolen fallen filamentartige Strukturen auf. Daneben sind aber oft auch granuläre und vesikuläre Anteile zu erkennen (Abb. 2 a).

Ncben den unregelmäßig geformten, amoeboiden Kriechformen finden sich immer wieder abgekugelte Zellen, die eine unterschiedlich stark ausgebildete Pellicula besitzen (Abb. $1 \mathrm{c}+2 \mathrm{c}$ ). Bei den Praecysten ist diese Pellicula nur schwach ausgebildet, und mit Glasmessern noch schneidbar (Ultramikrotomie). Im Gegensatz zur Kriechform erscheint bei der Praecyste das Cytoplasma wesentlich dichter. Stark entwickelt sind zudem die Golgikomplexe, die zum Teil in breiten Bahnen bis zur Zellmembran ziehen.

Zur Ultrastruktur der Dauercyste - der Überdauerungs- und Vermehrungsform (?) (Schulz-LANGNER, 1964) - erlauben unsere Untersuchungen keine Aussagen. Zum einen kommt es bei der Ultramikrotomie, bedingt durch die Härte der Pellicula der Cyste, fast stets zu Zerreißungen, zum anderen sind die cytoplasmatischen Anteile nur ungenügend fixiert, um Einzelheiten erkennen zu lassen. Offensichtlich ist das von uns angewandte wässrige Fixierungsmittel nicht in der Lage, die derbe Pellicula in ausreichend großer Geschwindigkeit zu durchdringen, um die lytischen Prozesse im Cysteninneren zu verhindern.

\section{DISKUSSION UND SCHLUSSFOLGERUNG}

Die am Aufbau der imaginalen Malpighischen Gefäße von Apis mellifera beteiligten Zellen sind über die gesamte Länge der Nierenorgane gleichgestaltet, wobei zwischen zwei verschiedenen Zelltypen - Haupt- und Nebenzellen unterschieden werden muß, die nebeneinander vorkommen. Den Gefäßen fehlt ganz offensichtlich eine durch stärker differenzierte Zelltypen bedingte regionale Gliederung, wie sie für verschiedene, insbesondere larvale Gefäße anderer Insekten beschrieben wird (Wigglesworth, 1931; Wigglissworth und Salpeter, 1962 ; Bacceti, Mazzi und Massimello, 1963 ; Berkaloif , 1960 ; Smith und Littau, 1960 ; Wessing und Eichelberi, 1969, 1972 a, b, 1975 ; EICHEL berG und Wessing, 1971, 1975 ; Eichelber;, 1979). Bei regional gegliederten MG kommt den verschiedenen Abschnitten auch eine unterschiedliche Bedeutung in der Harnbereitung zu, d.h. sie sind in unterschiedlich starkem Maße an Filtrations-, Sekretions-und Resorptionsprozessen beteiligt. Eine im Verlauf vom distalen Anfang zur proximalen Mündung der Gefäße in den Verdauungstrakt sich ändernde Funktion der Epithelzellen liegt bei der Honigbiene offensichtlich nicht vor. Das einheitliche Strukturbild der Zellen deutet auf gleiche Funktion der Zellen über die gesamte Länge der Schläuche hin. Dennoch ist auch bei 


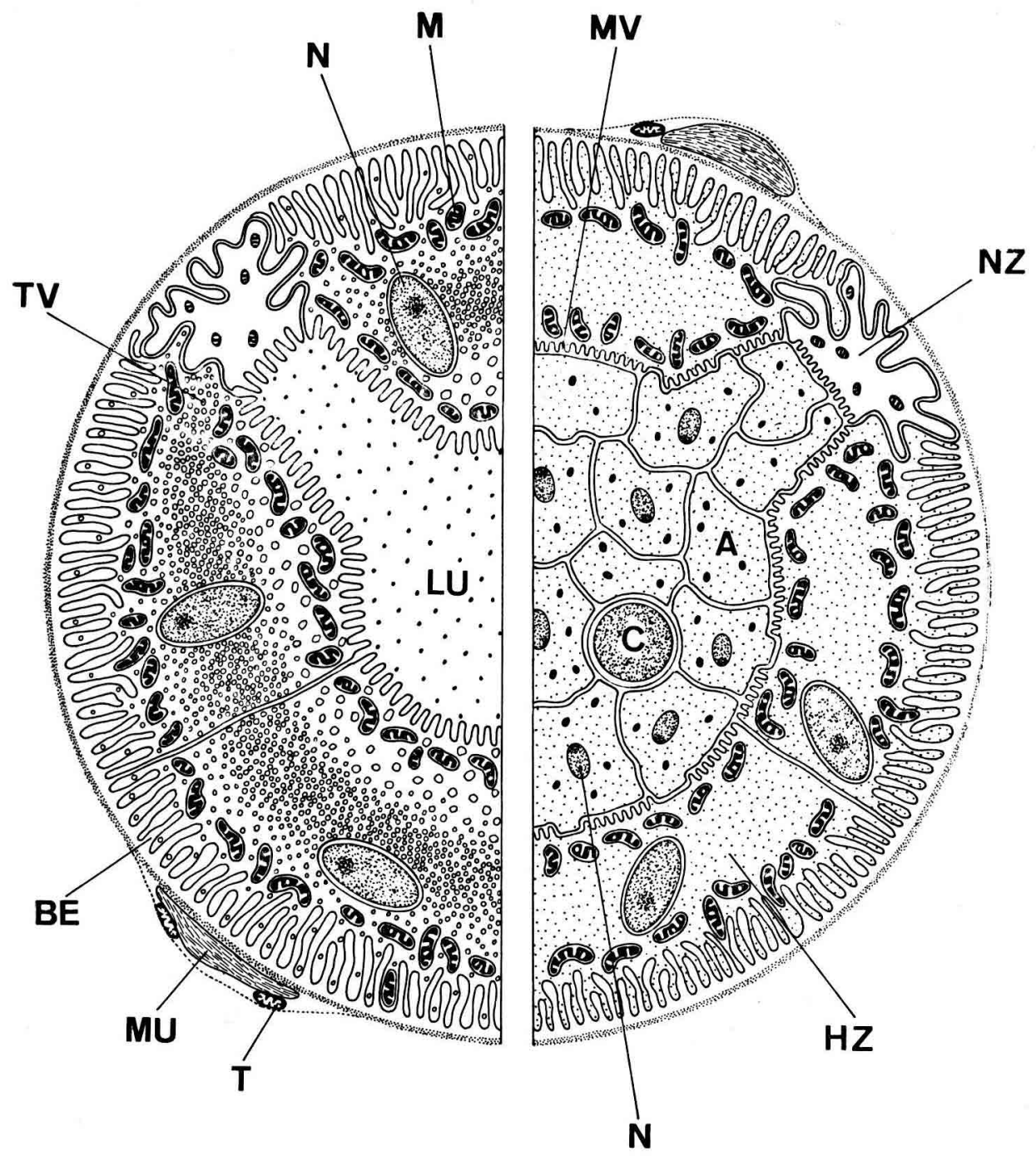

ABB. 4. Schematische Darstellung der Struktur eines Malpighischen Gefäßes von Apis mellifera in normalen (links) und im Malpighamoeba mellificae infizierten Zustand (rechts).

A - Amoeben, BE - Basale Zellmembraneinfaltungen, C - Cyste, HZ - Hauptzelle, LU - Gefäßlumen, M - Mitochondrium, MU - Muskelfaser, MV - Mikrovilli, N - Nucleus, NZ - Nebenzelle, T - Trachee, TV - Transpo:tvesikel.

FIG. 4. Diagram illustrating the ultrastructure of a Malpighian tube of Apis mellifera before (left) and after infection with Malpighamoeba mellificae (right).

A - amoeba, BE - basal infoldings, C - cyst, HZ - primary cell, LU - lumen, M - mitochondrion, MU - muscular fibre, MV - microvillus, $N$ - nucleus, $\mathrm{NZ}$ secondary sell, $\mathrm{T}$ - trachea, TV - transport vesicle. 
diesen Organen durchaus eine Arbeitsteilung nicht auszuschließen. So könnten simultan unterschiedliche Stofftransportprozesse - sogar in entgegengesetzten Richtungen - über die unterschiedlich strukturierten Haupt- und Nebenzellen ablaufen. Die physiologische Bedeutung der Nebenzellen ist bisher keineswegs erwiesen. BerRIDGE und OSchman (1969) vermuten für die, den Nebenzellen von Apis entsprechenden «stellate cells» von Calliphora erythrocephala, eine Bedeutung bei der selektiven transzellulären Ionenpassage. Auch das elektronenmikroskopische Bild gibt zu erkennen, daß sowohl die Nebenzellen, als auch insbesondere die Hauptzellen zu hoher Transportleistung in der Lage sind : sie zeigen den typischen Aufbau transportaktiver Zellen (BERridge und Oschman, 1972).

Wie wir bisher wissen, bestehen bei Insekten kaum Druckunterschiede zwischen der Leibeshöhle und dem Lumen der Malpighischen Gefäße (HeverT, 1975). Ein Druckgradient, wie er zum Beispiel für die Primärharnbildung in der Vertebratenniere verantwortlich ist, kann daher für den glcichen Vorgang bei Insekten keine Bedeutung haben. Aus Untersuchungen an Drosophila ist bekannt, daß eine Primärharnbildung hier über cine aktive Vesikulation und anschließende transzelluläre Passage zum Gefäßlumen erfolgt. Damit ist sicher auch die große. Menge an Vesikeln in den Hauptzellen der nicht infizierten Malpighischen Gefäße der Honigbiene zu erklären. Die Abnahme der Anzahl dieser Vesikel von basal nach apikal, und die Größenzunahme der Vesikel in gleicher Richtung. ergibt sich offensichtlich durch Fusion von mehreren kleineren Vesikeln im Verlauf ihrer transzellulären Passage. Bei den larvalen Gefäßen von Drosophila ist ein derartiger Vesikeltransport und damit gleichzeitig der Ort der Primärharnbildung auf den Anfangsbereich der Gefäße begrenzt. Bei Apis scheint nach den vorliegenden Befunden eine Primärharnbildung über die gesamte Länge der Gefäße zu erfolgen. Für die Bildung des Endharnes, d.h. für Rückresorption von für den Körper noch wertvollen Stoffen, sowie von Ionen und Wasser, ist bei der Biene offensichtlich der Enddarmabschnitt von großer Bedeutung. Insbesondere sind hierbei die Epithelien der Rektalpapillen zu nennen, deren Zellen ebenfalls aufgrund ihres ultrastrukturellen Aufbaues zu hoher Transportarbeit befähigt sind (Kümmel und Zerbst-BoroffKa, 1974).

Betrachtet man die Veränderungen an den Zellen der Malpighischen Gefäße der Honigbiene nach Infektion mit Malpighamoeba, so ist auffällig, daß sichtbare Strukturveränderungen, insbesondere an den für die Transportarbeit der Zellen wichtigen Organellen, auftreten : die zum Gefäßlumen gerichtete apikale Membranfläche des Mikrovillisaumes wird stark reduziert, die Mikrovilli werden zum Teil sogar vollständig eingeschmolzen. In einigen Amoebenzellen finden sich größere membranumhüllte Areale, die filamentartige Strukturen sowie granuläre und vesikuläre Anteile beinhalten.

Diese unsere Befunde werden gestützt durch lichtmikroskopische Unter- 
suchungen anderer Autoren (MAASSEN, 1919 ; Prell, 1926 ; FYG, 1932 ; GiORDani, 1959 ; Schulz-Langner, 1964 ; Cantwell, 1974).

Noch stärker wird aber nach Amoebeninfektion die Anzahl der Transportvesikel der Zellen reduziert, was zwangsläufig bedeutet, daß diese Gefäße für eine Harnproduktion kaum noch geeignet sind. Wir glauben daher, daß eine wichtige pathogene Wirkung der Malpighamoeben auf den gesamten Bienenorganismus in einer Unterbindung des Primärharnflusses beruht, der sekundär zu Vergiftungserscheinungen führen kann, die bis zum Tod der Tiere führen können. Die von Borchert (1966) beschriebenen Krankheitssymptome des Befalls durch Malpighamoeba mellificae, wie Anschwellung des Abdomens und ruhrartiger Durchfall, wurden bei den untersuchten Bienen nicht festgestellt.

Als weiterer Unterschied zwischen gesunden und infizierten Gefäßen ist die bereits lichtmikroskopisch sichtbare deutliche Abflachung der Epithelzellen mit Amoeben besiedelter Nierenorgane auffällig (FYG, 1932 ; GIORDANI, 1959 ; Schulz-Langner, 1964 ; Cantwell, 1974). Ob diese starke Abflachung der Epithelzellen nun rein mechanisch durch den hohen Binnendruck der sich teilenden amoeboiden Kriechformen zu erklären ist — wie es FYG (1932) annimmt - darüber erlauben auch unsere statischen EM-Aufnahmen keine Aussage. Sicher aber führt das mit $M$. mellificae erfüllte Lumen zu einer starken Beeinträchtigung des Harnabflusses, und trägt so zusätzlich zum Erliegen der exkretorischen und osmoregulatorischen Funktion der Malpighischen Gefäße bei.

Prell (1926, 1927), Giordani (1959) und Steinhaus (1963) beschreiben aufgrund lichtmikroskopischer Befunde noch erheblich weitergehende Veränderungen nach Amoebenbefall. Sie beschreiben eine Zerstörung des Cytoplasmas, sowie Strukturveränderungen am Zellkern und dessen teilweises Auseinanderbrechen. Derartig weitreichende Schädigungen konnten wir aufgrund unserer elektronenmikroskopischen Befunde nicht bestätigen. Insbesondere ein Aufplatzen der Nuclei erscheint uns unwahrscheinlich.

Eingegangen im Januar 1984. Angenommen im Oktober 1984.

\section{DANKSAGUNG}

Für die Hilfe beim Auffinden infizierter Tiere danken wir Herrn Imkermeister E. BREMER, für technische Unterstützung Frau A. Hudel. 


\title{
RESUME
}

\author{
ETUDE AU MICROSCOPE ELECTRONIQUE DE LA PARASITOSE \\ A MALPIGHAMOEBA MELLIFICAE (RHIZOPODA) DES TUBES DE MALPIGHI \\ D'APIS MELLIFICA
}

On a étudié l'ultrastructure des tubes de Malpighi d'Apis mellifica avant et après l'infection par Malpighamoeba mellificae. Les insectes ont d'abord été immoblisés par le froid. Les tubes ont été fixés par un mélange tamponné $(\mathrm{pH}=7,2)$ de tétroxyde d'osmium à $1 \%$ et colorés à l'acétate d'uranyle et à l'acide phosphotungstique. Le matériel a été inclus dans du Vestopal et coupé à l'ultratome III (LBK). On a pris des microphotos avec un EM 9 (Zeiss) et un EM 300 (Philips).

Les tubes ont une forme constante sur toute leur longueur et les cellules épithéliales révèlent la structure des cellules de transport. Le plasmalemme de base forme de nombreuses invaginations. Les mitochondries se trouvent particulièrement dans les régions basale et apicale. A l'apex les cellules forment une bordure en brosse dense (Fig. 1).

A côté de ces cellules dénommées primaires, sont également présentes des cellules individuelles ayant une architecture divergente (cellules secondaires). Elles semblent plus plates et leurs invaginations basales sont moins prononcées. Leurs mitochondries plus petites sont distribuées dans toute la cellule.

L'infection des tubes de Malpighi par Malpighamoeba mellificae provoque de nets changements dans la structure de la cellule. Toutes les cellules apparaissent nettement aplaties et la bordure en brosse a une taille fortement réduite. Les vésicules de transport séc:étrices, présentes en grand nombre dans les organes sains, sont totalement absentes. Ces variations, visibles au microscope électronique, permettent de conclure que le dégât primaire causé à l'insecte hôte est un fonctionnement défectueux du rein (Fig. 2 et 4 ).

Malpighamoeba mellificae apparât dans les tubes examinés sous forme amiboïde, sous forme de pré-kyste et de kyste permanent. On n'a pas trouvé de forme ciliée. On trouve la forme amiboïde à l'intérieur du lumen dilaté des tubes, disposée le plus souvent l'une contre lautre formant comme un pseudo-épithélium. Les kystes permanents sont des cellules arrondies, entourées d'une forte pellicule. Les pré-kystes ne possèdent pas une telle pellicule (Fig. 3).

\section{SUMMARY}

\section{ELECTRON MICROSCOPIC INVESTIGATIONS OF PARASITOSIS OF MALPIGHIAN TUBULES OF APIS MELLIFERA BY MALPIGHAMOEBA MELLIFICAE (RHIZOPODA)}

The ultrastructure of the Malpighian tubules of Apis mellifera was studied before and after the infection with Malpighamoeba mellificae.

At first the animals were immobilized at a low temperature. Tubules were fixed in buffered $1 \%$ osmium tetroxide ( $\mathrm{pH}$ 7.2) and stained with uranyl acetate and phosphotungstic acid. The material was embedded in Vestopal and cut with an Ultratom III (LKB). Micrographs were taken with an EM 9 (Zeiss) and an EM 300 (Philips).

The tubules are of uniform shape throughout their length, and the epithelial cells display the structure of transporting cells. The basal plasmalemma forms a great number of infoldings. The mitochondria are to be found especially in the basal and apical region. A dense brush border (Fig. 1) forms at the apex the cells.

Beside these so-called primary cells single cells with a divergent architecture are also present (secondary cells). These have a flatter appearance and their basal infoldings are not so strongly pronounced. Their smaller mitochondria are distributed over the entire cell. 
The infection of the Malpighian tubules with Malpighamoeba mellificae leads to marked changes in cell structure. All cells appear distinctly flattened and the brush border is significantly reduced in size. The secretory transport vesicles, which in healthy organs exist in great number, are completely absent. These variations, which can be perceived with an electrone microscope, allow one to conclude that the primary harm done to the host insect consists of an osmoregulatory malfunction (Fig. $2+4$ ).

Malpighamoeba mellificae appears in the tubules examined as an amoeboid form, as a pre-cyst and as a permanent-cyst. Ciliated forms were not found. The amoeboid form is found inside the expanded lumen of the tubules, mostly lying close together as a pseudo-epithelium. The permanent cyst are rounded cells, enclosed by a sturdy pellicula. The pre-cysts lack such a pellicula (Fig. 3).

\section{LITERATURVERZEICHNIS}

Bacetti B., Mazzi V. \& Massimelio G., 1963. - Richerche istochimiche c al microscopio elettronico sui tubi Malpighiani di Dacus oleae Gmel. II. L, Adulto. Redia, 48, 47-68.

Berkaloff M.A., 1960. - Contribution à l'étude des tubes de Malpighi et de l'excrétion chez. les insectes. Observation au microscope électronique. Ann. Sci. natur. Zool. Ser. XII, 2, 869-947.

Berridge M.I. \& Oscuman J.L., 1969. - A Structural Basis for Fluid Secretion by Malpighian Tubules. Tissue of Cell, Vol. 1, Nr. 2.

Berridge M.I. \& Oschman J.L., 1972. - Transporting Epithelia. New York - Iondon.

Borchert A., 1966. - Die Krankheiten und Schüdlinge der Honigbiene. - Leipzig, S. Hirzel, 190-201.

Cantwell G.E., 1974. - Insect Diseases, Vol. 2. - New York, Marcel Dekker.

Eicheiberg D. \& WEssing A., 1971. - Elektronenoptische Untersuchungen an den Nierentubuli (Malpighische Gefälıe) von Drosophila melanogaster. II. Transzelluläre membrangebundene Transportmechanismen. Z. Zellforsch, 121, 127-152.

Eichelberg D., \& Wessing A., 1975. - Morphology of the Malpighian Tubules of Insects. Fortschr. Zool., Bd. 23, Heft $2 / 3$.

Eichelberg D., 1979. - Feinstruktur und experimentelle Beeinflußbarkeit Malpighischer Gefäße bei Larven der Salzfliege Ephydra riparia. Entomol. Gen., 5 (4) : 301-315, Stuttgart-New York.

FYG W., 1932. - Beobachtungen über die Amoeben-Infektion der Malpighischen Gefäße bei der Honigbiene. Schweiz. Bienenztg., 55, 562-572 u. 609-616.

Giordani G., 1959. - Amoeba Disease of the Honey Bee, Apis mellifera Linnaeus, and an attempt at its Chemical Control. J. Insect Pathol, 1, 245-269.

Hevert F., 1975. - Physiologische Mechanismen bei der Harnbildung durch Malpighische Gefäße. Fortschr. Zool., 23, 173-192.

KüMMEL G. \& ZeRBST-BorofFKa J., 1974. - Elektronenmikroskopische und physiologische Untersuchungen an den Rectalpolstern von Apis mellifica. Cytobiologie, Bd. 9 (3), 432-459.

MaAssen A., 1919. - Weitere Mitteilungen über Bienenkrankheiten und ihre Bekämpfung. Mitt. aus der Biol. Reichsanstalt Land- und Forstwirtsch., Heft 17, 37-45.

Prell H., 1926-27. - Beiträge zur Kenntnis der Amoebenseuche der erwachsenen Honigbiene. Arch. Bienenkd., 7, 113-121.

Schulz-Langner E., 1957, - 1957 ein Amoebenjahr! Die Bienenzucht, 10, 147-149.

Schulz-Langner E., 1964. - Der Entwicklungsgang der in den Malpighischen Gefäßen der Honigbiene lebenden Amoebe (Malpighamoeba mellificae, Prell) in Kulturen und im Bienenkörper. Z. Bienenforsch., 7, Heft 1, 1-24. 
Schuiz-Langner E., 1969. - Untersuchungen über Anwesenheit und Verbrcitung des Amoebenparasiten (Malpighamoeba mell.) in einem Bienenvölkchen von April-Juli 1967. Z. Bienenforsch., 9, Heft 9, 381-389.

Smith D.S.d LrTiau V.C., 1960. - Cellular specialisation in the excretory cpithelia of an insect, Macrosteles fascifrons Stael (Homoptera). J. biophys, biochem. Cytol., 8, 103-133.

Steinhaus E.A., 1963. - Insect Pathology, Vol. 2. - London, New York, Academic Press, 348351 and $458-460$.

Wessing A. E Eicherberg D., 1969. - Elektronenoptische Untersuchungen an den Nierentubuli von Drosophila melanogaster. Z. Zellforsch., 101, 285-322.

WESSING A. \& EICHELBERg D, 1972 a. - Elektronenmikroskopische Untersuchungen an den Nierentubuli (Malpighische Gefäße) von Drosophila melanogaster III. Die intrazelluläre Speicherung der Aminosäure 3-Hydroxy-Kynurenin. Z. Zellforsch., 125, 132-142.

Wessing A. \& Eicher berg D., 1972 b. - Elektronenmikroskopische Untersuchungen an den Nierentubuli (Malpighische Gefäße) von Drosophila melanogaster IV. Die Lokalisation von Natriumund Chloridionen. Z.Zellforsch., 131, 269-286.

Wessing A. \& Eichelberg D., 1975. - Ultrastructural aspects of transport and accumulation of substances in the Malpighian tubules. Fortschr. Zool., 23, 148-172.

Wessing A. \& Eichisberg D., 1978. - Malpighian Tubules, Rectal papillae and Excretion. In Ashburner M. \& Wright T.R.F. Ed., Biology of Drosophila, Vol. 2 c, New York.

Wigglesworth V.B., 1931. - The physiology of excretion in a blood-sucking insect, Rhodnius prolixus (Hemiptera, Reduviidae) 11. Anatomy and histology of the excretory system. J. exp. Biol., 8, 428-442.

Wigglesworth V.B. \& Salpeter M.M., 1962. - Histology of the Malpighian tubules of Rhodnius prolixus Stal (Hemiptera). J. Insect Physiol., 8, 299-307.

Wohlfahrt-BottermanN K.E., 1957. - Die Kontrastierung tierischer Zellen und Gewebe im Rahmen ihrer elektronenmikroskopischen Untersuchung an ultradünnen Schnitten. Naturwissenschafien, 44, 287-288. 